\title{
A Review on Self-Cleaning Coatings for Tunnels
}

\author{
Yu Han*, Ang Zhan \\ CCCC First Highway Consultants Co., Ltd., Xi'an 710000, Shanxi Province, China \\ *Corresponding author: Yu Han, bby1124@163.com
}

Copyright: ( $) 2022$ Author(s). This is an open-access article distributed under the terms of the Creative Commons Attribution License (CC BY 4.0), permitting distribution and reproduction in any medium, provided the original work is cited.

\begin{abstract}
Self-cleaning coatings for tunnels can effectively remove dust and stains accumulated over the surface of tunnel linings and their appurtenances due to the closed environment and poor ventilation. This paper systematically introduces the current research status of self-cleaning coatings for tunnels, focusing on the development of super-hydrophobic self-cleaning coatings, superamphiphobic self-cleaning coatings, exhaust gas degradation coatings, fire retardant coatings, and tunnel deicing coatings. The advantages and disadvantages of the five functional coatings are then briefly described, and the problems of self-cleaning coatings for tunnels at the present stage are pointed out. Finally, the development direction of self-cleaning coatings for tunnels is proposed to provide a reference for the research and application of self-cleaning coatings for tunnels.
\end{abstract}

Keywords: Tunnel coatings; Self-cleaning; Super-hydrophobic; Superamphiphobic; Fire retardant; Exhaust gas degradation; De-icing

Online publication: January 12, 2022

\section{Introduction}

With the rapid development of a comprehensive transportation system in China, the construction of tunnels has also made great progress. People are becoming more concerned about the cleanliness of tunnels. On the one hand, the environment of a tunnel is relatively closed and poorly ventilated, which makes it difficult to exhaust car emissions in a timely manner, thus increasing the content of toxic and harmful substances, polluting the air and causing harm to personal health, reducing visibility, and hindering traffic safety. On the other hand, the dust, moisture, and stains on the wall surface are not cleaned in time, leading to the growth of microorganisms and bacteria as well as affecting the environmental hygiene. Therefore, there is an urgent need to develop coating materials for tunnels with self-cleaning function, which is of great significance to improving the traffic safety and environmental hygiene in tunnels ${ }^{[1,2]}$.

Self-cleaning coatings can remove surface pollutants by gravity, wind, and other physical means or remove dirt by photocatalysis and other chemical degradation processes. The use of these coatings does only lower the maintenance costs, but also significantly diminish environmental pollution. Therefore, selfcleaning coatings have application prospects in construction, transportation, and new energy industries. Currently, there are five types of self-cleaning coatings for tunnels: superhydrophobic self-cleaning coatings, superamphiphobic self-cleaning coatings, exhaust gas degradation coatings, fire retardant coatings, and de-icing coatings ${ }^{[3]}$. The research on superhydrophobic coatings originated from the selfcleaning properties of lotus leaves. Superhydrophobic coatings were developed based on the special microstructure and surface material of the lotus leaf surface, and superhydrophobic coatings were the first to be successfully applied in engineering construction. With the development of China's economy and the increasing traffic in tunnels as well as people's demand for environmental protection, superhydrophobic 
coatings with a single function can no longer meet the development needs. On this basis, superamphiphobic coatings and self-cleaning coatings with the function of exhaust gas degradation came into being. Several scholars have added flame retardant and high-temperature resistance materials to self-cleaning coatings for tunnels and successfully paired self-cleaning coatings with flame retardancy and fireproof properties. This has provided some guarantee for the safety of traffic in tunnels. The common de-icing coating is mostly used in highway and railroad traffic tunnels in the north of China. It effectively reduces the adhesion of ice in the tunnel during winter and ensures the safety of vehicle passage in winter. After years of research by experts at home and abroad, although self-cleaning coatings have achieved certain research results, there are still certain limitations in large-scale engineering applications, thus requiring further research and improvement.

\section{Research status at home and abroad}

\subsection{Superhydrophobic self-cleaning coating}

Superhydrophobic coatings are the most widely used in tunnel self-cleaning coatings. These coatings are prepared by organically combining the free energy of the wet surface and the surface microstructure of the solid surface. Superhydrophobic coatings can be divided into inorganic and organic-inorganic hybrid according to their material compositions.

Inorganic superhydrophobic coatings are generally made from inorganic raw materials to form rough structures with low surface energy and hydrophobic surfaces. The chemical modification to reduce the surface energy is carried out after constructing a micro- and nanostructured rough surface on the hydrophilic substrate. Tao Defu prepared superhydrophobic coatings with rough structure using glass as substrate, which has excellent self-cleaning properties, chemical resistance, and adhesion between the coating and substrate ${ }^{[1]}$. Bao Xiaohui used electrochemical etching to construct micro-nano bilayer structures on the surface of silicon carbide (SiC) particle composites with static contact angles up to $160.7^{\circ}$ and superhydrophobic effect ${ }^{[4]}$.

Organic-inorganic hybrid superhydrophobic coatings are generally organic solutions with surface modification to achieve special surface microstructures and reduce the surface energy. By adding hydrophobic nano-SiO 2 , Huang Shuo modified the common PRTV silicone rubber coating to produce a superhydrophobic coating with a water contact angle of $150^{\circ}$, as shown in Figure 1(a). In a certain range, as the amount of $\mathrm{SiO}_{2}$ nanoparticle additions increases, the more uniformly the micro-nano structure is dispersed on the surface of the coating, and the better the hydrophobic properties are (Figure 2) ${ }^{[5]}$. Pi Ke prepared an organic-inorganic hybrid self-cleaning coating for tunnels by using free radical polymerization reaction to prepare silicone emulsions and hybridizing them with silica sol and titanium sol. The results showed that the contact angle of the coating is between $50^{\circ}$ and $60^{\circ}$, as shown in Figure 1(b), and the coating has good water resistance as well as solubility resistance. Moreover, the coating did not delaminate, blister, or peel after 24 hours of immersion ${ }^{[6]}$. 
(a)

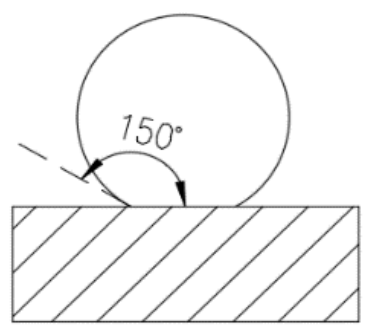

Figure 1. Contact angle of different coatings with water; (a) Contact angle of $\mathrm{SiO}_{2}$-modified PRTV silicone rubber coating; (b) Contact angle of titanium hybrid silicone acrylic emulsion coating

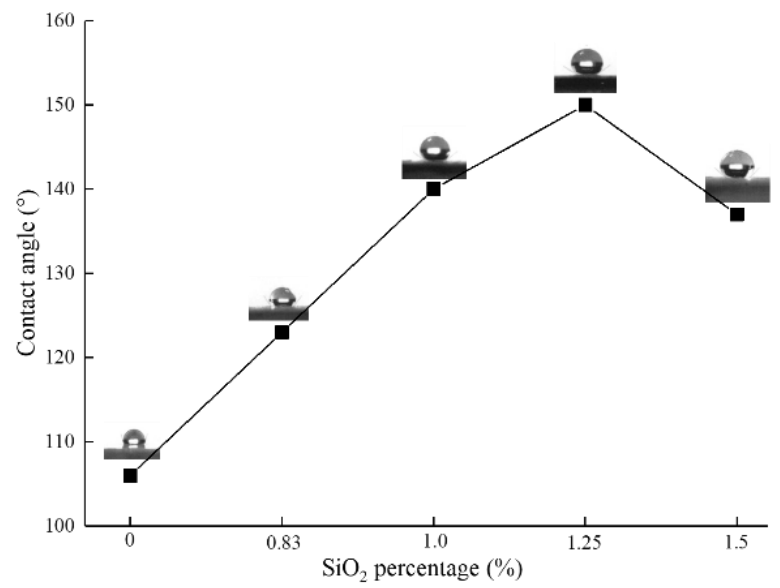

Figure 2. Influence of $\mathrm{SiO}_{2}$ content on the contact angle of PRTV silicone rubber coating

\subsection{Superamphiphobic self-cleaning coating}

Compared with superhydrophobic coatings, superamphiphobic coatings are hydrophobic and oleophobic, in which they can adapt to harsher environments. Therefore, the surface energy of superamphiphobic coatings is lower, the preparation process is more complicated, and the cost is higher, but its application in the field of tunnel self-cleaning is more extensive. Yao Daozhou prepared a water-based superamphiphobic self-cleaning coating for tunnels via a two-step wet chemical method, and he found that the coating can maintain superamphiphobic performance when exposed to high temperatures as high as $150^{\circ} \mathrm{C}$ for 48 hours, and it does not peel easily, only at $-20^{\circ} \mathrm{C}$. The superhydrophobic properties remained even after the coating was immersed in acid and alkali with $\mathrm{pH}$ values of 1-13 and in $\mathrm{NaCl}$ solution at $1 \mathrm{~mol} / \mathrm{L}$ for 2 hours. Through that, it can be appreciated that it has good acid and alkali resistance ${ }^{[7]}$.

He Sizhe prepared transparent hydrophobic and oleophobic coating materials using organic resin hybridized with nano- $\mathrm{SiO}_{2}$ as the substrate layer and then modified with a solution of low surface energy ${ }^{[8]}$. Based on aqueous superhydrophobic coatings, Yabin Li prepared a perfluorodecyl polysiloxane (fluoroPOS) modified $\mathrm{SiO}_{2}\left(\mathrm{SiO}_{2} @\right.$ Fluoro-POS) suspension via hydrolysis and condensation of perfluorodecyltriethoxysilane and tetraethoxysilane (TEOS). Upon processing, a $\mathrm{PU} / \mathrm{SiO}_{2} @ \mathrm{Fluoro} \mathrm{POS}$ superhydrophobic self-cleaning coating is obtained. The coating has excellent superhydrophobic properties, good mechanical, chemical, and thermal stability, as well as anti-icing properties ${ }^{[9]}$.

\subsection{Exhaust gas degradation coating}

With the increasing traffic volume, the pollution of tunnel environment caused by exhaust emissions from vehicles is becoming more and more serious, and the exhaust gas degradation coating is receiving much 
more attention. Currently, photocatalysts are mainly used to clear exhaust emissions in tunnels.

Guo Yinchuan used modified nano photocatalytic coating on the surface of tunnel asphalt mixture. When the doping amounts of nano- $\mathrm{TiO}_{2}$ and nano- $\mathrm{CeO}_{2}$ are $5 \%$ and $10 \%$, respectively, the peak degradation efficiency can be obtained; the degradation efficiencies of hydrocarbon (HC) and nitric oxide (NO) are $41.7 \%$ and $68.7 \%$, respectively, while the exhaust gas degradation rates are $78.5 \mathrm{ppm} / \mathrm{min}$ and $333.9 \mathrm{ppm} / \mathrm{min}$, respectively ${ }^{[10]}$. Wang Tong prepared functional coatings by combining high alumina cement with thermal insulation as well as flame retardant materials, modified with nano- $\mathrm{TiO}_{2}$, vapor phase $\mathrm{SiO}_{2}$, and silicone water repellent. The scanning electron microscope (SEM) image is shown in Figure 3. Moreover, she found that the prepared coating has better fireproof performance, with catalytic degradation efficiencies of $23.4 \%, 2.5 \%$, and $2.9 \%$ for $\mathrm{NO}_{x}, \mathrm{CO}$, and $\mathrm{CO}_{2}$, respectively, in automotive exhaust gas under visible light for 60 minutes; the water droplet contact angle is $134.2^{\circ}$, and the coating has the functions of hydrophobic self-cleaning, fire protection, and exhaust gas degradation [11]. Xia Huiyun used titanium dioxide $\left(\mathrm{TiO}_{2}\right)$ as the photocatalytic material and aqueous emulsion as the coating film-forming substance. Flame retardants and self-cleaning additives were added into the coating composition to make functional tunnel coatings. The results showed that the coating has good adhesion and aging resistance, and the degradation efficiencies of $\mathrm{NO}_{x}, \mathrm{CO}$, and $\mathrm{CO}_{2}$ are $67.40 \%, 25.10 \%$, and $27.64 \%$, respectively, within 2 hours; it is able to withstand $300^{\circ} \mathrm{C}$ without being damaged at a coating thickness of $1 \mathrm{~mm}$, and the contact angle with water is up to $159^{\circ}{ }^{[12]}$.

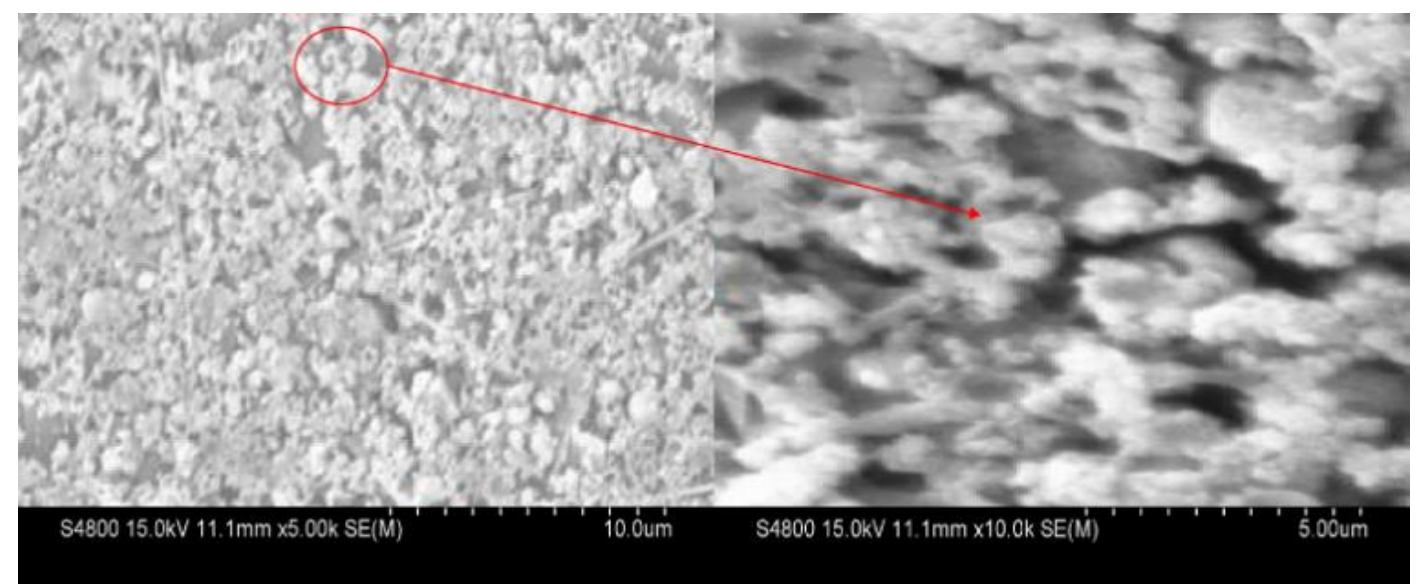

Figure 3. SEM image of functional coating

\subsection{Fire retardant coating}

Fireproof coating carries out various physical and chemical reactions in order to play a flame retardant role. The part of the coating that can play a fire retardant role is the flame retardant. Flame retardants are usually divided into two types: additive and reactive. Additive flame retardants are mostly inorganic flame retardants, halogen flame retardants, phosphorus flame retardants, and nitrogen flame retardants, which can be mixed with resin or binder and have a wide range of application. Reactive flame retardants are mostly organic halogen and phosphorus monomers with reactive functional groups, which can react with polymer compound chains and have good durability as well as less adverse effects on material performance.

Xiang Qingsong calculated the temperature field of a tunnel under the heating condition of hydrocarbon fire by optimizing the ratio of raw materials and using the finite element analysis software, ANSYS. He found the order of influence of each material on the fire resistance limit of fireproof coating: composite binder $>$ foaming material $>$ inorganic thermal insulation filler ${ }^{[13]}$. Rasmika used phosphorus-based polyester polyol and 1,3,3-trimethyl-5-isocyanato-1-isocyanatomethyl cyclohexane for the synthesis of a flame retardant waterborne polyurethane coating. He found that the coating has good flame retardant effect 
with good thermal stability ${ }^{[14]}$. Yan synthesized phosphorus/boron-containing flame retardants from cyclic phosphate ester (PEA) and polyethylene glycol borate (PEG-BA) as well as added phosphorus-containing flame retardants to polyurethane to prepare transparent fireproof coatings ${ }^{[15]}$.

\subsection{De-icing coating}

At present, the main methods for railroad tunnel de-icing are thermal ice melting and manual icing. Thermal ice melting requires a large among of heat and power consumption, while manual icing involves high work intensity, harsh working environment, and great danger. Therefore, snow-melting agents and ice melting coatings came into being. However, most of the current snow-melting agents are expensive and have pollution and corrosion issues; in addition, there are only a few research on tunnel de-icing coating.

Superhydrophobic coating can reduce the bonding force between the lining surface and ice, based on which the problem of icing in tunnel vaults can be solved. Jiang Songli developed a binder coating containing cationic emulsified asphalt, which can slowly release snow-melting substances for this very purpose. It has been found that the resulting snow-melting coating has good de-icing performance and durability ${ }^{[16]}$. Li Na simulated a test in the laboratory by applying Wearlon Super F-1 for de-icing in low temperature tunnels. It has been found that the coating does not only have a strong de-icing effect, but also excellent properties against water, sand, chemical solvent, salt, as well as low and high temperatures, which would effectively solve the safety problem of railroad tunnel vault during winter due to icing ${ }^{[17]}$. Zhao Shurui created a porous epoxy resin substrate using the respiration diagram method and prepared the coating by polydimethylsiloxane modification and dimethyl silicone oil lubricating fluid infusion. The coating has good adhesion properties on different substrates, and the infused coating has a low roll angle. It can be used for the control of ice adhesions in tunnels, exhibiting low ice adhesion strength and delayed icing time with excellent anti-icing performance ${ }^{[18]}$.

\section{Conclusion and prospects}

(1) At present, the research on superhydrophobic self-cleaning coatings for tunnels is relatively comprehensive, but there is still some room for further progress, such as cost reduction and durability enhancement.

(2) Most of the superhydrophobic and oleophobic coating materials do not have high oleophobic contact angles and cannot really achieve the superhydrophobic effect; these materials are limited by their preparation process and cost. Therefore, further work should be done to improve the oleophobicity by adjusting and optimizing the rough structure or by further reducing the surface energy of the coating.

(3) There are still some problems with exhaust gas degradation coatings for tunnels. On the one hand, most of the exhaust gas degradation coatings require photocatalysis. However, the tunnel environment is usually closed with insufficient natural light; in that case, the photocatalytic efficiency is low. On the basis of exhaust gas degradation and self-cleaning, multifunctional exhaust gas degradation coatings with self-cleaning properties for tunnels can be developed.

(4) The development and application of fireproof coatings for tunnels started late. Although domestic and foreign scholars have done much research on it, the fire resistance limit, bond strength, and other properties still require improvement. Further improving the fire resistance limit, bond strength, and other properties of fireproof coatings is still the focus and difficulty of current research. 
(5) The research on de-icing coating is still in the preliminary exploration stage, and the research reports related to this topic are relatively rare. However, this coating is known to be environmental-friendly, convenient, and efficient, thus having large room for research.

\section{Funding}

This work was financially supported by National Key R\&D Program of China (2018YFB1600101).

\section{Disclosure statement}

The authors declare that there is no conflict of interest.

\section{Author contributions}

Han Yu conceived the idea of the study, Zhan Ang performed the experiments, and Han Yu analyzed the data as well as wrote the paper.

\section{References}

[1] Tao D, 2020, Preparation and Properties of Epoxy-Based Super-Hydrophobic Coatings. Hubei University of Technology.

[2] Zhuang L, Li J, Liu W, et al., 2020, Preparation and Performance Evaluation of a New Durable SuperHydrophobic Wall Coating. Surface Technology, 12(10): 1-10.

[3] Lan M, Wen Q, Zhu J, 2020, Research Progress on the Preparation and Application of Self-Cleaning Coatings. Materials Protection, 53(03): 129-134.

[4] Bao X, Ming P, Bi X, 2016, Preparation of Superhydrophobic Surface Based on SiC Particulate Reinforced Composite. Journal of Inorganic Materials, 31(4): 383-387.

[5] Huang S, 2012, Study of Ice-Melt Type Anti-Ice Coating. Wuhan University of Technology, Hubei.

[6] Pi K, Wang Z, Xiang J, et al., 2019, Study on Preparation and Properties of Organic Inorganic Hybrid Self-Cleaning Coating. New Building Materials, 46(3): 49-52.

[7] Yao D, 2019, Research on the Fabrication of Waterborne Superamphiphobic Coatings and Their Applications on Filter Materials. Southeast University.

[8] He S, 2018, Preparation of Environment-Friendly Super-Hydrophobic Coating. Xiamen University.

[9] Li Y, 2019, Preparation and Properties of Environmentally Friendly Waterborne Super-Hydrophobic and Superamphiphobic Coatings. Lanzhou University of Technology.

[10] Guo Y, Xie Z, Shen A, et al., 2019, Research on Tunnel Tail Gas Degradation of Coated Composite Nano-Photocatalytic Materials. Bulletin of the Chinese Ceramic Society, 38(08): 2563-2569.

[11] Wang T, 2017, Preparation and Performance of Multifunctional Nanocoatings for Long and Large Tunnels. Nanjing Forestry University.

[12] Xia H, Zhao X, Liu G, et al., 2019, Preparation and Properties of Self-cleaning Flame Retardant Coatings for Automobile Exhaust Degradation by Visible Light Photocatalysis. Bulletin of the Chinese Ceramic Society, 38(03): 589-595.

[13] Xiang Q, 2013, Study on Composite Tunnel Fire Retardant Coating and Its Properties. Hunan University, Hunan. 
[14] Patel R, Kapatel P, 2018, Waterborne Polyurethanes: A Three Step Synthetic Approach Towards Environmental Friendly Flame Retardant Coatings. Progress in Organic Coatings, 125(2018): 186-194.

[15] Yan L, Xu ZS, Nan D, 2019, Effects of Polyethylene Glycol Borate on the Flame Retardancy and Smoke Suppression Properties of Transparent Fire-Retardant Coatings Applied on Wood Substrates. Progress in Organic Coatings, 135(2019): 123-134.

[16] Jiang S, 2012, Research on Environmentally Friendly Asphalt Pavement Ice and Snow Melting Coating. Chang'an University, Shaanxi.

[17] Li N, 2019, Study of Tunnel De-Icing Coating. Technology Wind, 2019(13): 93.

[18] Zhao S, Shen T, Li Y, et al., 2021, Anti-Icing Coating of Epoxy Resin-Based Super-Slip Liquid Infusion Based on Respiration Diagram Method. Acta Polymerica Sinica, 52(10): 1053-1057.

Publisher's note

Bio-Byword Scientific Publishing remains neutral with regard to jurisdictional claims in published maps and institutional affiliations. 